\title{
An Adaptive Fuzzy Sliding Mode Control Design for a Class of Uncertain Horizontal Platform Systems
}

\author{
Wei Xiang and Xiejin Liu \\ Department of Mathematics and Computer Science, Huainan Normal University, Huainan 232038, China \\ Correspondence should be addressed to Wei Xiang; xiangwei27@126.com
}

Received 6 January 2014; Revised 29 January 2014; Accepted 13 February 2014; Published 20 March 2014

Academic Editor: Gani Stamov

Copyright (c) $2014 \mathrm{~W}$. Xiang and X. Liu. This is an open access article distributed under the Creative Commons Attribution License, which permits unrestricted use, distribution, and reproduction in any medium, provided the original work is properly cited.

\begin{abstract}
This paper presents an adaptive fuzzy sliding mode control design for a class of uncertain horizontal platform systems (HPSs). Firstly, a nonsingular terminal sliding surface is proposed for HPSs. Then, a fuzzy logic system is introduced to estimate the system uncertainties. The adaptive fuzzy sliding mode controller can guarantee the stability of the closed-loop system. The corresponding numerical simulations are demonstrated to verify the effectiveness of the proposed method.
\end{abstract}

\section{Introduction}

Over the past two decades, many mechanical systems with chaotic phenomena have been developed $[1,2]$. One of the most interesting and attractive nonlinear dynamical systems is the horizontal platform system (HPS). It is a mechanical device that can freely rotate around the horizontal axis. The horizontal platform devices are widely used in earthquake engineering and offshore. In [3], the horizontal platform system displays diverse chaotic behavior. So, how to suppress chaotic phenomenon is a hot research topic. Until now, a wide variety of approaches have been proposed for stabilizing chaotic systems such as adaptive control $[4,5]$, observerbased control [6], sliding mode control [7, 8], backstepping control $[9,10]$, and fuzzy control $[11,12]$. In [3], two identical HPSs can be synchronized by using linear, sinusoidal, and exponential state error feedback controllers. Wu et al. [13] proposed a sufficient criterion for global chaos synchronization between two identical HPSs coupled by using linear state error feedback controller. Meanwhile, they achieved the robust synchronization of the chaotic HPS with phase difference and parameter mismatches in [14]. Based on the Lyapunov stability theorem and Sylvester criterion, some algebraic sufficient criteria for synchronization of two HPSs coupled by sinusoidal state error feedback control have been derived in [15].

Sliding mode control (SMC) has been studied extensively for over 50 years and widely used in practical applications due to its simplicity and robustness against parameter variations and disturbances. Despite the extensive research activities carried out, the key technical problems associated with SMC remain as challenging research questions due to demands for new industrial applications and technological advances. Yu and Kaynak [7] provided the state of the art of recent developments in SMC systems with soft computing (SC) and examined key technical research issues and future perspectives. For the horizontal platform system, Pai and Yau [16] proposed an integral-type sliding mode controller for generalized projective synchronization of two HPSs with uncertainties.

However, most of the above-mentioned methods have been proposed to synchronize two identical HPSs asymptotically. Nevertheless, from a practical engineering point of view, it is more reasonable to stabilize the HPS in finite time under the affection of uncertainties. In order to achieve finite time stabilization, the terminal sliding mode (TSM) concept has been proposed [17]. TSM is a fast response control scheme. The advantage of using TSM is that the states of the controlled system can reach zero in a finite time. For the HPS nonlinear system, how to design the TSM is still an open problem. Fuzzy control schemes have been found to be particularly useful to model unknown functions in nonlinear systems rather than only unknown parameters. There have been significant research efforts on adaptive fuzzy control for nonlinear systems. For example, 


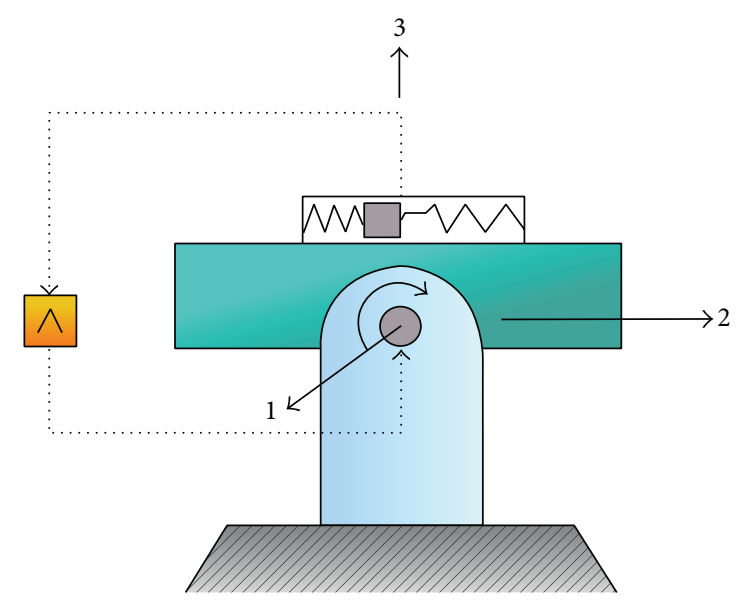

Figure 1: Physical model of the horizontal platform system.

Tong and Li [18] developed the stable adaptive fuzzy sliding mode controller for nonlinear multivariable systems with unavailable states. Very recently, M. P. Aghababa and H. P. Aghababa [19] proposed adaptive controllers to achieve finite time synchronization of two nonautonomous chaotic HPSs. However, the bounds of uncertainties of HPSs are assumed to be known. In practical engineering, we could not obtain the bounds of the uncertainties. In order to overcome this limitation, we employ fuzzy system to approximate the HPS's unknown nonlinear functions and adaptive laws are derived based on Lyapunov stability analysis for online updating the parameter of the model. So, the major contribution of this paper is that a nonsingular terminal sliding mode control scheme incorporating fuzzy control technique is proposed to stabilize uncertain HPS.

The organization of this paper is described as follows. In Section 2, system model is derived, and the problem statement is also given. In Section 3, the design of the proposed control strategies is discussed. The simulation results are presented to demonstrate the effectiveness of proposed control scheme in Section 4. Conclusion is presented in Section 5 .

\section{Description of HPS Dynamics and Problem Statement}

The HPS is a mechanical device composed of a platform and an accelerometer located on the platform (see Figure 1). The platform can freely rotate about the horizontal axis, which penetrates its mass center. The accelerometer produces an output signal to the actuator, subsequently generating a torque to inverse the rotation of the platform to balance the HPS, when the platform deviates from horizon. The motion equations of the HPS are given by [18]

$$
A \ddot{y}+D \dot{y}+k g \sin y-\frac{3 g}{R}(B-C) \cos y \sin y=F \cos \omega t,
$$

where $y$ is the rotation of the platform relative to horizon, $A, B$, and $C$ are inertia moment of the platform, $D$ is the

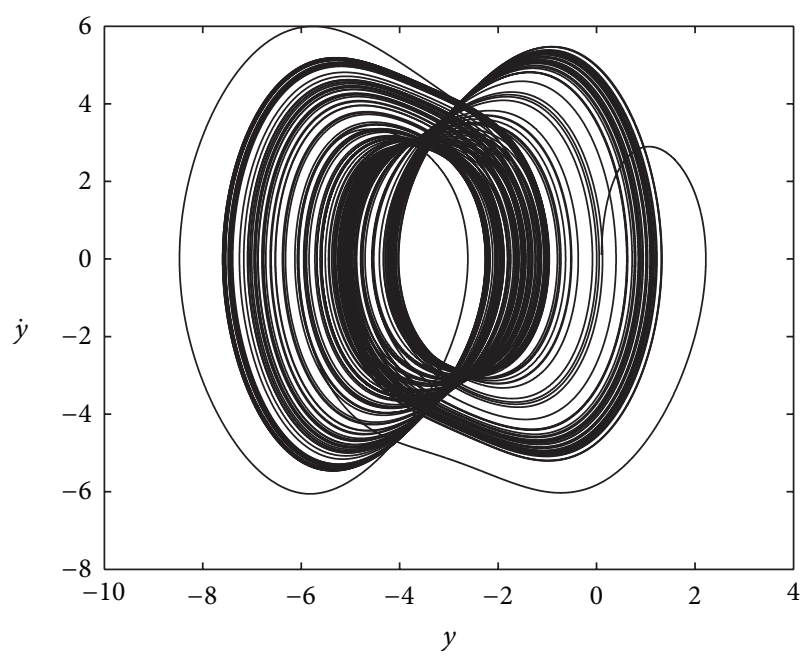

Figure 2: Phase plane trajectory of system (1).

damping coefficient, $k$ is the proportional constant of the accelerometer, $g$ is the acceleration constant of gravity, $R$ is the radius of Earth, and $F \cos \omega t$ is the harmonic torque. System (1) exhibits chaotic behavior with $A=0.3, B=0.5$, $C=0.2, k=0.11559633, R=6378000, F=3.4$, and $\omega=1.8$ (see Figure 2).

For simplicity, we introduce the following notations: $x=$ $\left[x_{1}, x_{2}\right]^{T}=[y, \dot{y}]^{T}$; then the dynamic model of (1) with unknown disturbances can be described by the following equations:

$$
\begin{gathered}
\dot{x}_{1}=x_{2}, \\
\dot{x}_{2}=f(x, t)+d(t, x)+u(t),
\end{gathered}
$$

where $x$ is the state vector which is assumed available for measurement, $u(t) \in R$ is the control input, and $d(t, x)$ is unknown external disturbance. Consider that $f(x, t)=$ $-a x_{2}-b \sin x_{1}+l \cos x_{1} \sin x_{1}+h \cos \omega t$ is assumed to be unknown, $a=D / A, b=\mathrm{kg} / A, l=3 g(B-C) / R A$, and $h=F / A$. 


\section{Adaptive Fuzzy TSM Control Design}

In order to design an adaptive fuzzy terminal sliding mode controller for the system (1), the following terminal sliding surface is defined [8]:

$$
s(t)=x_{2}+x_{1}+x_{1}^{\alpha}=0,
$$

where $0<\alpha=q / p<1$ and $q, p>0$ are odd integers. Then, for any initial value of the state $x(t)$ at $t=0$, the solution of (3) will reach $s=0$ in finite time and $t_{s}=(1 /(1-\alpha)) \ln x(0)^{1-\alpha}+1$. Notice that, for $\alpha x_{1}^{\alpha-1} x_{2}$, due to $q-p<0$, singularity will occur as $x_{1}=0$ and $x_{2} \neq 0$. To avoid the singularity problem and guarantee that the system (2) will reach the sliding mode surface (3) in finite time, we redefine $\alpha x_{1}^{\alpha-1} x_{2}$ as follows:

$$
\alpha x_{1}^{\alpha-1} x_{2}= \begin{cases}\alpha x_{1}^{\alpha-1} x_{2}, & \text { if } x_{1} \neq 0, x_{2} \neq 0, \\ \alpha \epsilon^{\alpha-1} x_{2}, & \text { if } x_{1}=0, x_{2} \neq 0, \\ 0, & \text { if } x_{1}=0, x_{2}=0,\end{cases}
$$

where $\epsilon$ is a small positive constant. To implement the TSM controller, we define the following TSM-type teaching law:

$$
\dot{s}(t)=-k_{1} s-k_{2} e^{-\lambda t} \frac{s}{|s|^{\beta}},
$$

with $k_{1}>0, k_{2}>0, \lambda>0$, and $\beta>0$. Differentiating (3) gives

$$
\dot{s}(t)=x_{2}+\alpha x_{1}^{\alpha-1} x_{2}+F(t, x)+u(t) .
$$

And according to (2) and (5), the equivalent control law can be given by

$$
\begin{aligned}
u_{\mathrm{eq}}(t)= & -x_{2}-\alpha x_{1}^{\alpha-1} x_{2}-F(t, x) \\
& -k_{1} s-k_{2} e^{-\lambda t} \frac{s}{|s|^{\beta}},
\end{aligned}
$$

where $F(t, x)=f(t, x)+d(t, x)$. Owing to $f(t, x)$ and $d(t, x)$ being unknown, so $F(t, x)$ is unknown. Thus, the control law (7) is usually difficult to be obtained. Here, we use fuzzy logic system to approximate the nonlinear unknown function.

3.1. Fuzzy Approximator. The fuzzy IF-THEN rules are used to perform a mapping from an input vector $\mathbf{x}=$ $\left[x_{1}, x_{2}, \ldots, x_{n}\right]^{T} \in R^{n}$ to an output $y \in R$. The $r$ th fuzzy rule is written as $R^{r}$ : if $x_{1}$ is $A_{1}^{r}\left(x_{1}\right)$ and... and $x_{n}$ is $A_{n}^{r}\left(x_{n}\right)$, then $y$ is $B^{r}$, where $A^{r}$ and $B^{r}$ are fuzzy sets with membership functions $\mu_{A_{i}^{r}}\left(x_{i}\right)$ and $\mu_{B_{i}^{r}}(y)$, respectively, and $\mathbf{x}$ belongs to a compact set. If we use the product-inference rule, singleton fuzzifier, and center-average defuzzifier, then the output of fuzzy logic system can be defined as

$$
y=\frac{\sum_{i=1}^{n_{r}} \tilde{y}^{i}\left(\prod_{j=1}^{n} \mu_{A_{j}^{i}}\left(x_{j}\right)\right)}{\sum_{i=1}^{n_{r}}\left(\prod_{j=1}^{n} \mu_{A_{j}^{i}}\left(x_{j}\right)\right)}=\eta^{T} \Psi(\mathbf{x}),
$$

where $n_{r}$ is the number of total fuzzy rules, $\tilde{y}^{i}$ is the point at which $\mu_{B_{i}^{r}}\left(\tilde{y}^{i}\right)=1, \mu_{A_{j}^{i}}\left(x_{j}\right)$ is the membership function of the fuzzy variable $x_{j}$ characterized by Gaussian, $\eta=$ $\left[\tilde{y}^{1}, \tilde{y}^{2}, \ldots, \widetilde{y}^{n_{r}}\right]^{T}$ is an adjustable parameter vector, and $\Psi=$ $\left[\psi^{1}, \psi^{2}, \ldots, \psi^{n_{r}}\right]^{T}$ is a fuzzy basis vector, where $\psi^{i}$ is defined as

$$
\psi^{i}(\mathbf{x})=\frac{\prod_{j=1}^{n} \mu_{A_{j}^{i}}\left(x_{j}\right)}{\sum_{i=1}^{n_{r}}\left(\prod_{j=1}^{n} \mu_{A_{j}^{i}}\left(x_{j}\right)\right)} .
$$

3.2. Adaptive Fuzzy Terminal Sliding Mode Controller Design. So, by applying the introduced fuzzy systems in (8), approximation of function $F(t, x)$ can be expressed as follows:

$$
\begin{gathered}
\widehat{F}\left(x, \eta_{f}\right)=\left[\widehat{f}_{1}\left(x, \eta_{f_{1}}\right), \widehat{f}_{2}\left(x, \eta_{f_{2}}\right)\right]^{T}, \\
\widehat{f}_{i}\left(x, \eta_{f_{i}}\right)=\eta_{f_{i}}^{T} \psi_{f_{i}}(x),
\end{gathered}
$$

where $\eta_{f_{i}}^{T}$ is adjustable parameter vectors, $i=1$, 2. Optimal parameter $\eta_{f_{i}}^{*}$ can be defined such that

$$
\eta_{f_{i}}^{*}=\arg \min _{\eta_{f_{i}}}\left\{\sup _{x \in D_{x}}\left|f_{i}(t, x)-\widehat{f}_{i}\left(x, \eta_{f_{i}}\right)\right|\right\},
$$

where $D_{x}$ is an allowable set of the state vector. And minimum estimation error can be expressed as

$$
\varepsilon_{f}(t, x)=F(t, x)-F^{*}\left(x, \eta_{f}^{*}\right) .
$$

It is assumed that minimum estimation errors are bounded for all $x \in D_{x}:\left|\varepsilon_{f}(t, x)\right| \leq \varepsilon ; \varepsilon$ is positive constant.

According to (7), (10) can be written as

$$
u_{\text {equ }}=-x_{2}-\alpha x_{1}^{\alpha-1} x_{2}-\widehat{F}\left(x, \eta_{f}\right)-k_{1} s-k_{2} e^{-\lambda t} \frac{s}{|s|^{\beta}} .
$$

And the proposed controller is designed as

$$
u=u_{\text {equ }}+u_{r}
$$

with $u_{r}=-\widehat{\varepsilon} \operatorname{sign}(s) ; \widehat{\varepsilon}$ is an estimate of $\varepsilon$.

To generate the approximation $F(t, x)$ and $\varepsilon$ online, we choose the following adaptation laws:

$$
\begin{aligned}
\dot{\eta}_{f_{i}} & =\lambda_{f_{i}} \psi_{f_{i}} s_{i}, \\
\dot{\widehat{\varepsilon}} & =\lambda_{\varepsilon}|s|,
\end{aligned}
$$

where $\lambda_{\varepsilon}>0$ and $\lambda_{f_{i}}>0, i=1,2$.

Theorem 1. Consider the uncertain horizontal platform system (2). If $F(t, \mathbf{x})$ is approximated by (10) and input controller and adaptive laws are selected as (10) and (14), respectively, then, all signals in the closed-loop system are bounded; $\lim _{t \rightarrow \infty} \eta_{f_{i}}=\eta_{f_{i}}^{*}$ and $\lim _{t \rightarrow \infty} \widehat{\varepsilon}=\varepsilon$.

Proof. Consider a Lyapunov function as

$$
V_{1}=\frac{1}{2} s^{2}+\frac{1}{2 \lambda_{\varepsilon}} \widetilde{\varepsilon}^{2}+\frac{1}{2} \sum_{i=1}^{2} \frac{1}{\lambda_{f_{i}}} \widetilde{\eta}_{f_{i}}^{T} \widetilde{\eta}_{f_{i}}
$$


where $\widetilde{\eta}_{f_{i}} \stackrel{\text { def }}{=} \eta_{f_{i}}^{*}-\eta_{f_{i}}$ and $\widetilde{\varepsilon} \stackrel{\text { def }}{=} \varepsilon-\widehat{\varepsilon}$. One can obtain the time derivative of (16) as

$$
\begin{aligned}
& \dot{V}_{1}=s\left(x+2+\alpha x_{1}^{\alpha-1}+F(t, x)+u\right) \\
& -\frac{\dot{\widetilde{\varepsilon}}}{\lambda_{\varepsilon}}-\sum_{i=1}^{2} \frac{1}{\lambda_{f_{i}}} \widetilde{\eta}_{f_{i}}^{T} \dot{\hat{\eta}}_{f_{i}} \\
& =s\left(F^{*}\left(x, \eta_{f}^{*}\right)+\varepsilon_{f}(t, x)-\widehat{F}\left(x, \eta_{f}\right)\right. \\
& \left.-k_{1} s-k_{2} e^{-\lambda t} \frac{s}{|s|^{\beta}}+u_{r}\right) \\
& -\frac{\dot{\mathcal{E}} \dot{\mathcal{\varepsilon}}}{\lambda_{\varepsilon}}-\sum_{i=1}^{2} \frac{1}{\lambda_{f_{i}}} \tilde{\eta}_{f_{i}}^{T} \dot{\hat{\eta}}_{f_{i}} \\
& \leq \sum_{i=1}^{2} \widetilde{\eta}_{f_{i}}^{T} \psi_{f_{i}} s_{i}+\varepsilon|s|-k_{1}|s|^{2}-k_{2} e^{-\lambda t}|s|^{2-\beta} \\
& -\widehat{\varepsilon}|s|-\frac{\dot{\mathcal{\varepsilon}}}{\lambda_{\varepsilon}}-\sum_{i=1}^{2} \frac{1}{\lambda_{f_{i}}} \widetilde{\eta}_{f_{i}}^{T} \dot{\hat{\eta}}_{f_{i}} .
\end{aligned}
$$

Substituting (15) into above inequality shows that

$$
\dot{V}_{1}<-k_{1}|s|^{2}-k_{2} e^{-\lambda t}|s|^{2-\beta}<0, \quad \text { for } s \neq 0 .
$$

Based on Lyapunov theory, one can show that all signals in the closed-loop system are bounded and $\eta_{f_{i}}$ and $\widehat{\varepsilon}$ converge to $\eta_{f_{i}}^{*}$ and $\varepsilon$, respectively, when $t \rightarrow \infty$. This completes the proof.

Lemma 2. If a Lyapunov function can be defined as

$$
\dot{V}(x)+a_{1} V(x)+a_{2} e^{-a_{3} t} \leq 0,
$$

where $a_{1}, a_{2}, a_{3}>0$, then the settling time is given by [20]

$$
t_{s} \leq \begin{cases}\frac{1}{a_{1}-a_{3}} \ln \left[1+\frac{a_{1}-a_{3}}{a_{2}} V\left(x_{0}\right)\right], & \text { if } a_{1} \neq a_{3} ; \\ \frac{V\left(x_{0}\right)}{a_{2}}, & \text { if } a_{1}=a_{3} .\end{cases}
$$

Theorem 3. If $\widehat{F}\left(x, \eta_{f}\right)=F^{*}\left(x, \eta_{f}^{*}\right)$, then terminal sliding mode surface $s=0$ will be reached in finite time.

Proof. For $\widehat{F}\left(x, \eta_{f}\right)=F^{*}\left(x, \eta_{f}^{*}\right)$, let $\bar{V}_{1}=(1 / 2) s^{2} ;$ then from (16), we have

$$
\dot{\bar{V}}_{1}=s \dot{s}=|\mathbf{s}| \frac{d|s|}{d t}<-k_{1}|s|^{2}-k_{2} e^{-\lambda t}|s|^{2-\beta} .
$$

So, we get

$$
\frac{d|s|}{d t}<-k_{1}|s|-k_{2} e^{-\lambda t}|s|^{1-\beta}
$$

Denoting $w=|s|^{\beta}$, from (22), the time derivative of $w$ yields

$$
\frac{d w}{d t}=\beta|w|^{\beta-1} \frac{d|s|}{d t}=-\beta k_{1} w-k_{2} \beta e^{-\lambda t} .
$$

According to Lemma 2, integral terminal sliding mode surface $s=0$ will be reached in finite time. This completes the proof.

Remark 4. If we select another fast TSM manifold: $s=x_{2}+$ $x_{1}+\xi \operatorname{sign}\left(x_{1}\right)$, we can obtain similar results.

Remark 5. The characteristics of the proposed TSM include the following: (i) the finite convergence time can be easily adjusted according to (3) and (4); (ii) the singular problem does not occur on the control law.

\section{Simulation Studies}

In this section, the numerical simulations are performed to verify and demonstrate the effectiveness of the proposed control scheme. Firstly, we show the method in [20]. Define sliding proportional + integral + derivative (PID) surface as follows:

$$
\bar{s}=k_{3} y+k_{4} \int_{0}^{t} y(\tau) d \tau+k_{5} \dot{y},
$$

where the control gains $k_{3}, k_{4}$, and $k_{5}$ are properly chosen such that the characteristic polynomial in $k_{3} \dot{y}+k_{4} y+k_{5} \ddot{y}=0$ is strictly Hurwitz. Assume $F(t, x)$ is bounded by $|F(t, x)| \leq$ $D^{*}, D^{*}$ is unknown positive constant, and $x=[y, \dot{y}]^{T}$. The control law is designed as

$$
\begin{gathered}
u=-\frac{k_{3}}{k_{5}} \dot{y}-\frac{k_{4}}{k_{5}} y-\widehat{D} \operatorname{sign}(\bar{s})-k_{6} \operatorname{sign}(\bar{s}), \\
\dot{\hat{D}}=k_{3}|\bar{s}|,
\end{gathered}
$$

where $\widehat{D}$ is an estimate of $D^{*}$ and $k_{j}$ is designed positive constant, $j=3,4,5,6$. We chose parameters $A=0.3, B=0.5$, $C=0.2, D=0.4, k=0.11559633, g=9.8, R=6378000$, $F=3.4, \omega=1.8, I_{\alpha}=0.061 \mathrm{~kg} \mathrm{~m}^{2}, k_{3}=5, k_{4}=6, k_{5}=1$, and $k_{6}=2$. The initial conditions $\left[\begin{array}{ll}y & \dot{y}\end{array}\right]^{T}=\left[\begin{array}{ll}0.1 & 0.1\end{array}\right]^{T}$ and the external disturbance $d(t, x)=0.2 \sin 0.2 y$. Under the control law (25), Figures 3 and 4 show the time response of the state $[y, \dot{y}]^{T}$ and $u$, respectively.

Now, we chose parameters $k_{1}=3, k_{2}=4, \alpha=3 / 5, \epsilon=$ $0.01, \beta=0.08, \lambda_{\varepsilon}=\lambda_{f_{i}}=2, i=1,2$, and $\lambda=3$. The Gaussian membership functions are assigned to $x_{i}$ over interval $[-6,6]$, and each of the fuzzy systems uses $7^{2}$ fuzzy rules to model $\widehat{F}\left(x, \eta_{f}\right)$ :

$$
\begin{gathered}
\mu_{A_{i}^{1}}=\exp \left[\frac{-(x+6)^{2}}{2}\right], \quad \mu_{A_{i}^{2}}=\exp \left[\frac{-(x+4)^{2}}{2}\right], \\
\mu_{A_{i}^{3}}=\exp \left[\frac{-(x+2)^{2}}{2}\right], \quad \mu_{A_{i}^{4}}=\exp \left[\frac{-(x+0)^{2}}{2}\right], \\
\mu_{A_{i}^{5}}=\exp \left[\frac{-(x-2)^{2}}{2}\right], \quad \mu_{A_{i}^{6}}=\exp \left[\frac{-(x-4)^{2}}{2}\right], \\
\mu_{A_{i}^{7}}=\exp \left[\frac{-(x-6)^{2}}{2}\right] .
\end{gathered}
$$




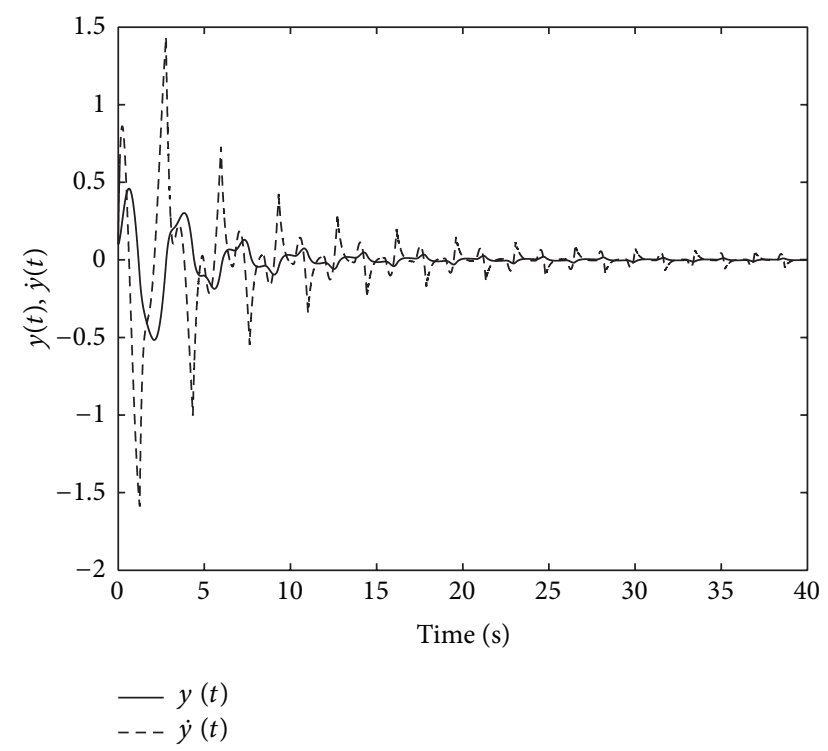

FIgURE 3: Time response $[y, \dot{y}]^{T}$ of system (1) under the method of [21].

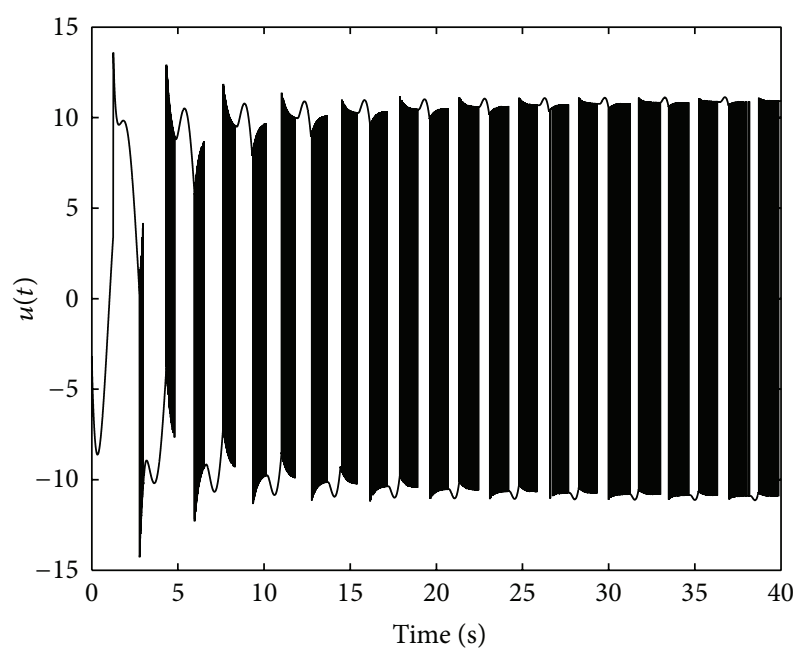

$-u(t)$

FIgURE 4: Time response $u(t)$ of system (1) under the method of [20].

The states of controlled system (1) are shown in Figure 5. One can see that the states are all driven to zero quickly by using the proposed adaptive terminal sliding mode control (13)-(15). Figure 6 displays the time response of $u(t)$. By comparison, we can conclude that it is an effective method proposed in this paper to reduce the chattering phenomenon. The robustness and stability of system are improved.

\section{Conclusion}

In this paper, an adaptive fuzzy terminal sliding mode control scheme has been proposed for HPSs. Based on

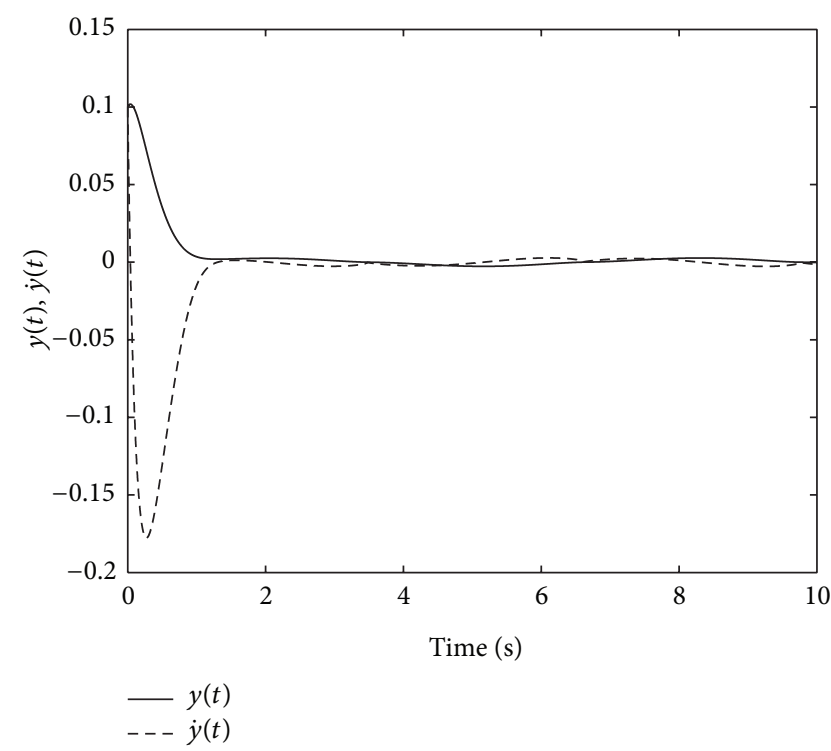

FIGURE 5: Time response $[y, \dot{y}]^{T}$ of system (1) with the control scheme (13)-(15).

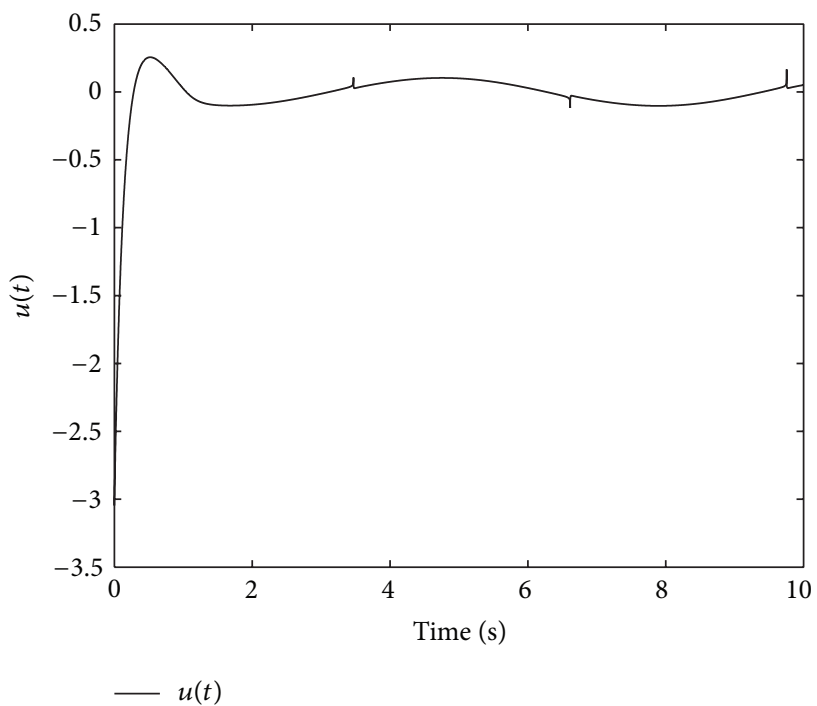

FIGURE 6: Time response $u(t)$ of system (1) with the control scheme (13)-(15).

fuzzy system rules, the proposed control approach guarantees the boundedness of all the signals in closed-loop system. Comparative examples have shown the effectiveness of the proposed method. Our next research direction is how to design finite time control scheme for uncertain HPS with unknown control gain.

\section{Conflict of Interests}

The authors declare that there is no conflict of interests regarding the publication of this paper. 


\section{Acknowledgments}

The authors gratefully acknowledge the support of the National Science Foundation of China (Grant no. 11172125) and Anhui Provincial Natural Science University research funding, China (KJ2012A257 and KJ2013A239).

\section{References}

[1] Y. Chen, X. Wu, and Z. Liu, "Global chaos synchronization of electro-mechanical gyrostat systems via variable substitution control," Chaos, Solitons and Fractals, vol. 42, no. 2, pp. 11971205, 2009.

[2] Z.-M. Ge and W.-R. Jhuang, "Chaos, control and synchronization of a fractional order rotational mechanical system with a centrifugal governor," Chaos, Solitons and Fractals, vol. 33, no. 1, pp. 270-289, 2007.

[3] Z.-M. Ge, T.-C. Yu, and Y.-S. Chen, "Chaos synchronization of a horizontal platform system," Journal of Sound and Vibration, vol. 268, no. 4, pp. 731-749, 2003.

[4] T. Shinbrot, E. Ott, C. Grebogi, and J. A. Yorke, "Using chaos to direct trajectories to targets," Physical Review Letters, vol. 65, no. 26, pp. 3215-3218, 1990.

[5] Z. Zhang, H. Shen, and J. Li, "Adaptive stabilization of uncertain unified chaotic systems with nonlinear input," Applied Mathematics and Computation, vol. 218, no. 8, pp. 4260-4267, 2011.

[6] M. Chen, D. Zhou, and Y. Shang, "A new observer-based synchronization scheme for private communication," Chaos Solitons and Fractals, vol. 24, no. 4, pp. 1025-1030, 2005.

[7] X. H. Yu and O. Kaynak, "Sliding-mode control with soft computing: a survey," IEEE Transactions on Industrial Electronics, vol. 56, no. 9, pp. 3275-3285, 2009.

[8] W. Xiang and Y. Huangpu, "Second-order terminal sliding mode controller for a class of chaotic systems with unmatched uncertainties," Communications in Nonlinear Science and Numerical Simulation, vol. 15, no. 11, pp. 3241-3247, 2010.

[9] C.-L. Chen, C. C. Peng, and H.-T. Yau, "High-order sliding mode controller with backstepping design for aeroelastic systems," Communications in Nonlinear Science and Numerical Simulation, vol. 17, no. 4, pp. 1813-1823, 2012.

[10] N. B. He, Q. Gao, and C. S. Jiang, "Robust adaptive control for a class of chaotic system using backstepping," Procedia Engineering, vol. 15, pp. 1229-1233, 2011.

[11] A. Boulkroune, M. Tadjine, M. M'Saad, and M. Farza, "Fuzzy adaptive controller for MIMO nonlinear systems with known and unknown control direction," Fuzzy Sets and Systems, vol. 161, no. 6, pp. 797-820, 2010.

[12] L. X. Wang, A Course in Fuzzy Ststems and Control, PrenticeHill, Englewood Cliffs, NJ, USA, 1997.

[13] X. Wu, J. Cai, and M. Wang, "Master-slave chaos synchronization criteria for the horizontal platform systems via linear state error feedback control," Journal of Sound and Vibration, vol. 295, no. 1-2, pp. 378-387, 2006.

[14] X. Wu, J. Cai, and M. Wang, "Robust synchronization of chaotic horizontal platform systems with phase difference," Journal of Sound and Vibration, vol. 305, no. 3, pp. 481-491, 2007.

[15] J. Cai, X. Wu, and S. Chen, "Chaos synchronization criteria and costs of sinusoidally coupled horizontal platform systems," Mathematical Problems in Engineering, vol. 2007, Article ID 86852, 10 pages, 2007.
[16] N.-S. Pai and H.-T. Yau, "Generalized projective synchronization for the horizontal platform systems via an integral-type sliding mode control," Journal of Vibration and Control, vol. 17, no. 1, pp. 11-17, 2011.

[17] S. P. Bhat and D. S. Bernstein, "Finite-time stability of continuous autonomous systems," SIAM Journal on Control and Optimization, vol. 38, no. 3, pp. 751-766, 2000.

[18] S. Tong and H.-X. Li, "Fuzzy adaptive sliding-mode control for MIMO nonlinear systems," IEEE Transactions on Fuzzy Systems, vol. 11, no. 3, pp. 354-360, 2003.

[19] M. P. Aghababa and H. P. Aghababa, "Finite-time stabilization of a non-autonomous chaotic rotating mechanical system," Journal of the Franklin Institute. Engineering and Applied Mathematics, vol. 349, no. 9, pp. 2875-2888, 2012.

[20] L. Liu, Z. Han, and W. Li, "Global sliding mode control and application in chaotic systems," Nonlinear Dynamics, vol. 56, no. 1-2, pp. 193-198, 2009.

[21] I. Eker, "Sliding mode control with PID sliding surface and experimental application to an electromechanical plant," ISA Transactions, vol. 45, no. 1, pp. 109-118, 2006. 


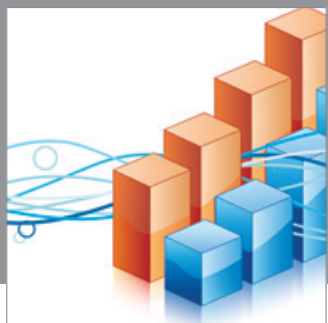

Advances in

Operations Research

mansans

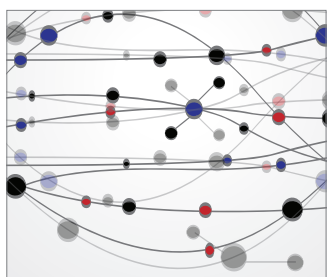

The Scientific World Journal
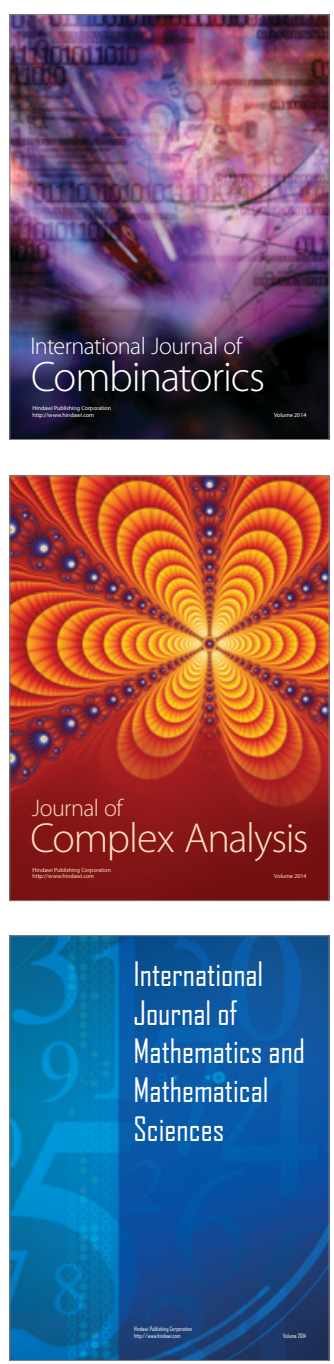
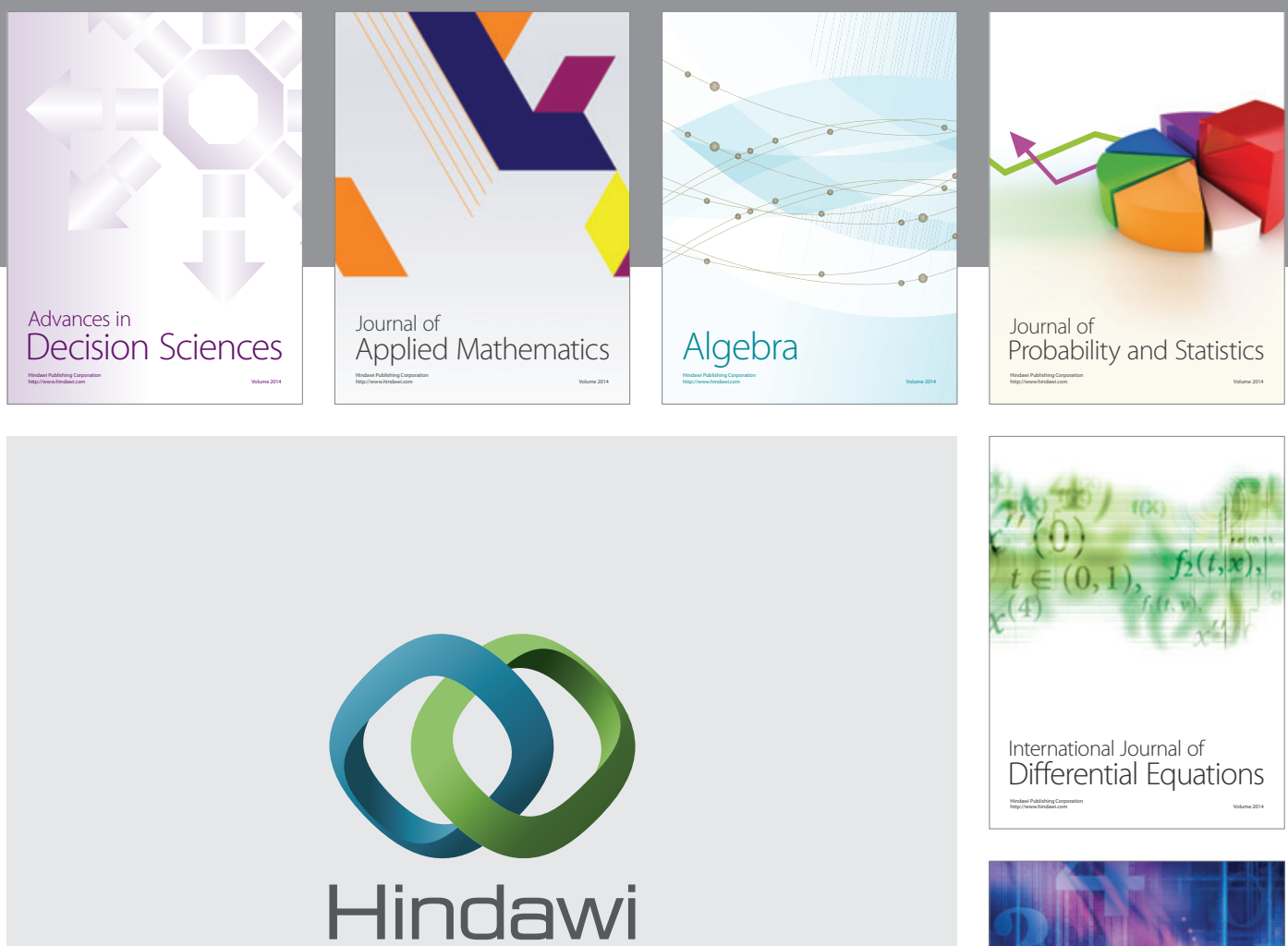

Submit your manuscripts at http://www.hindawi.com
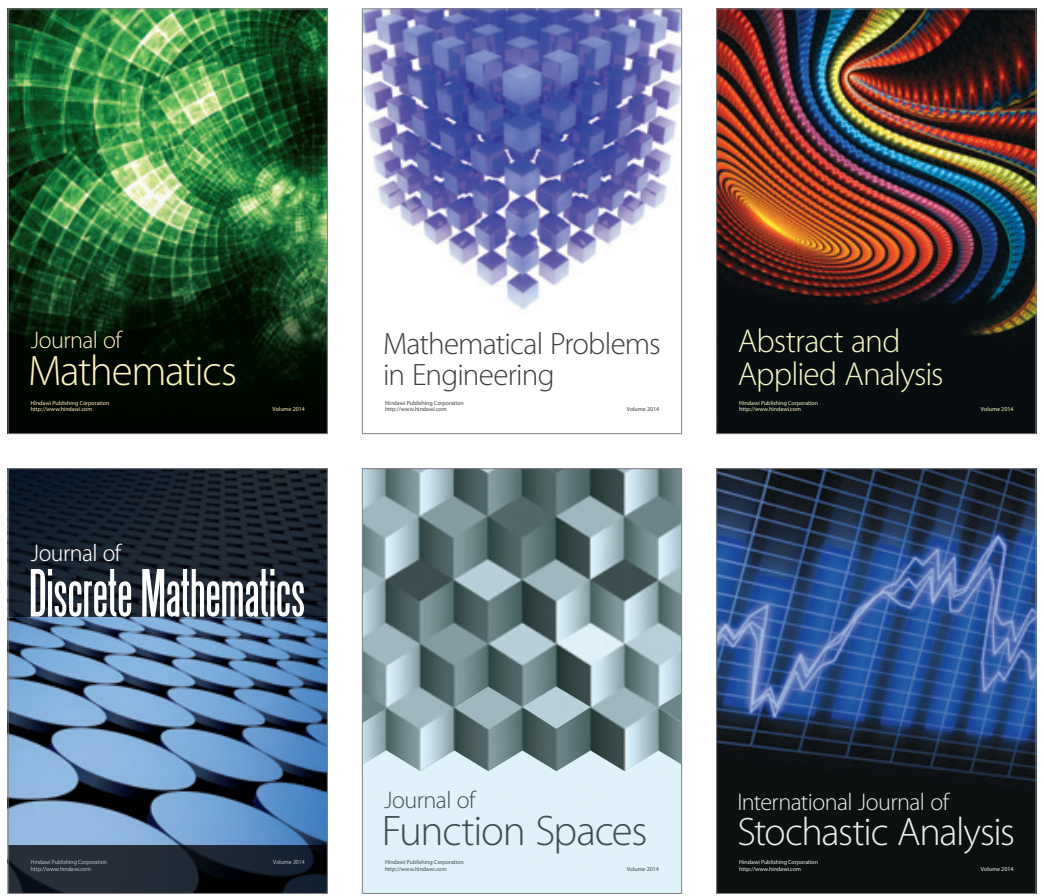

Journal of

Function Spaces

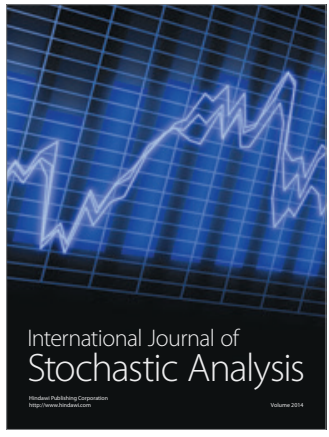

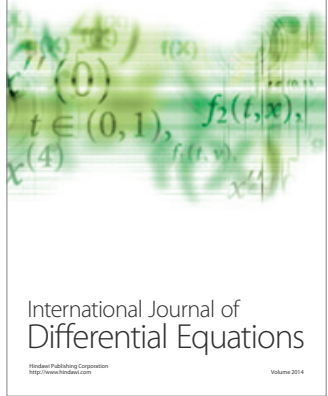
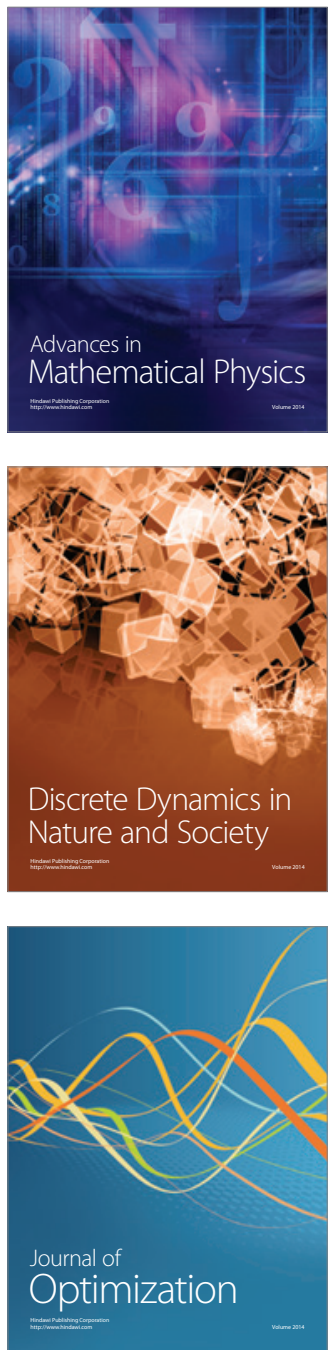